\title{
riccafd
}

Revista Iberoamericana de Ciencias de la Actividad Física y el Deporte

\section{SATISFACCIÓN DEL PROFESORADO DE EDUCACIÓN INFANTIL PARTICIPANTE EN UNA EXPERIENCIA DE APRENDIZAJE-SERVICIO BASADA EN RECREOS ACTIVOS}

SATISFACTION OF CHILDHOOD EDUCATION TEACHERS

WHO PARTICIPED IN A SERVICE-LEARNING EXPERIENCE BASED TO ACTIVE SCHOOL RECESSES

Sáez-Gallego, NM ${ }^{1 A C D}$, Abellán, J'BCF

${ }^{1}$ Sáez-Gallego, NM. Facultad de Educación de Toledo. Universidad de Castilla - La Mancha.

España. Nieves.Saez@uclm.es

${ }^{2}$ Abellán, J. Facultad de Educación de Cuenca. Universidad de Castilla - La Mancha.

España. Jorge.Abellan@uclm.es

Responsabilidades

A Diseño de la investigación

B Recolector de datos

C Redactor del trabajo

D Tratamiento estadístico

E Apoyo económico

$\mathrm{F}$ Idea original y coordinador de toda la investigación

Recibido el 5 de enero de 2020

Aceptado el 26 de febrero de 2020

Correspondencia: Nieves María Sáez Gallego. Nieves.Saez@uclm.es

DOI: http://dx.doi.org/10.24310/riccafd.2020.v9i1.8311

\section{RESUMEN}

El Aprendizaje - Servicio (ApS) es una metodología orientada a promover el aprendizaje de los estudiantes mediante la conexión entre las enseñanzas recibidas en la Universidad y el contexto real de aplicación de las mismas, a través de la prestación de un servicio a la sociedad. El presente trabajo evalúa la satisfacción de los maestros de Educación Infantil de diferentes centros educativos en los que se había aplicado una propuesta de ApS basada en recreos activos.

15 maestros de 8 centros educativos, presentes durante el desarrollo de la actividad, completaron una escala de valoración de la experiencia elaborada Ad-hoc. Esta se compuso de dos secciones: la primera recogía la valoración 
de la organización, el material, el disfrute, la participación y la novedad, utilizando una escala tipo Likert de 5 puntos; la segunda parte estaba formada por tres pregun-tas abiertas sobre los aspectos positivos, negativos y la intención de participar nuevamente en una experiencia similar.

Los resultados muestran que todos los aspectos evaluados obtienen puntuaciones cercanas al máximo posible. Los aspectos mejor valorados fueron la variedad de propuestas y su originalidad, así como la diversión y la implicación activa de los niños. El aspecto peor valorado fue la escasa duración de la propuesta. Todos los participantes estaban dispuestos a participar de nuevo en una experiencia similar.

A modo de conclusión, el ApS no sólo es una metodología atractiva para el alumnado de la Universidad, sino que permite prestar servicios de calidad, aspecto que queda reflejado en la satisfacción de los colectivos implicados.

Palabras clave: ambientes de aprendizaje, material reciclado, metodologías de enseñanza.

\section{ABSTRACT}

Service-Learning (SL) is a methodology aimed at promoting student learning through the connection between the teachings in the University and the real context of their application, by providing a service to society. The present work evaluates the satisfaction of the childhood education teachers of different educative centres in which a proposal of SL based on active recreation has been applied.

15 teachers from 8 educational centres, present during the development of the activity, completed an assessment scale developed Ad-hoc. This is composed of two sections: the first part recognizes the assessment of the organization, the material, the enjoyment, the participation and the novelty, using a 5-point Likert-type scale; the second part consisted of open questions about the positive and negative aspects and the intention to participate again in a similar experience.

The results show all aspects obtained scores close to the maximum possible. The aspects most valued were the variety of proposals and their originality, as well as the fun and active involvement of the children. The worst aspect was the duration of the proposal. All participants were willing to participate in a new one in a similar experience.

In conclusion, SL is not only an attractive methodology for students of the University, but also provides quality services, an aspect that is reflected in the satisfaction of the groups involved.

Key words: learning environments, recycled material, teaching methodologies. 


\section{INTRODUCCIÓN}

Una de las metodologías de enseñanza universitaria que más auge está teniendo en los últimos tiempos es el Aprendizaje - Servicio (ApS). Esta está orientada a promover el aprendizaje de los estudiantes mediante la conexión entre las enseñanzas recibidas en la Universidad y el contexto real de aplicación de las mismas. Su puesta en práctica se basa en la realización de un servicio a la sociedad, una vez detectada la necesidad de este, que permite al alumnado poner en práctica los contenidos teóricos adquiridos en la Universidad y tomar decisiones en una situación real.

El ApS ha sido utilizado en diferentes dominios para favorecer el desarrollo competencial del alumnado. Concretamente, ha sido utilizado en la formación de maestros de diferentes especialidades y etapas educativas ${ }^{1,2,7,8,11}$. La mayoría de los autores afirman que esta metodología motiva al alumnado hacia el aprendizaje, además de educar en habilidades sociales y valores. En concreto, se ha afirmado que esta metodología ayuda a crear futuros profesionales socialmente responsables, y que mejora el conocimiento de ellos mismos y de la diversidad ${ }^{2}$.

En el ámbito de la formación inicial del profesorado, el ApS ha sido considerado como muy relevante ${ }^{3}$, como es el caso de los futuros maestros de Educación Infantil (en adelante El incluidos en este trabajo, específicamente centrados en el trabajo de la EF en El. Existen algunos ejemplos en los que se ha combinado el ApS y la EF. Chiva-Bartoll, Peris y Piquer mostraron que un programa de ApS obtuvo mejoras en el concepto de personalidad eficaz de un grupo de futuros docentes de EF ${ }^{7}$. El ApS también se ha utilizado en EF con la intención de disminuir la exclusión social de los receptores del servicio. Por ejemplo, Vázquez, Liesa, y Lozano utilizaron un programa de recreos cooperativos realizado por futuros maestros de EF con el objetivo de reducir la exclusión social de los participantes, mientras los futuros maestros aprendían competencias transversales de su grado ${ }^{14}$. En definitiva, y de acuerdo con Capella, Gil, y Martí, entendemos que la aplicación del ApS en EF supone una práctica novedosa, y debido a las características específicas de la materia, eminentemente práctica, la EF es un marco ideal para la utilización de esta metodología ${ }^{5}$.

Entre las posibilidades de aplicación de esta metodología, en el presente trabajo se ha optado por utilizar el tiempo de recreo, espacio habitual de implementación de ApS, como ya se ha realizado en otros estudios ${ }^{14}$, y su combinación con los ambientes de aprendizaje. Un ambiente de aprendizaje es un espacio tan sugerente que por sí mismo capta la atención del alumnado para su utilización ${ }^{4}$ y que persigue un fin concreto, como el trabajo de un determinado contenido que surge a partir del juego libre de los participantes. Podríamos decir que los ambientes de aprendizaje son propuestas que utilizan materiales variados, que deberían ser atractivos y provocadores por sí mismos ${ }^{9}$. Aunque los ambientes de aprendizaje no son exclusivos del área de EF (se pueden consultar algunos ejemplos del empleo 
de los ambientes para el desarrollo de otros contenidos en el trabajo de Riera, Ferrer y Ribas $^{13}$ ), parece que son muy adecuados para el desarrollo de contenidos motrices. Son muchos los ejemplos de implementación de ambientes de aprendizaje para el trabajo de contenidos motrices, especialmente contenidos perceptivo-motrices. Por ejemplo, Nielsen, Romance y Chinchilla expusieron una propuesta de ambientes de aprendizaje en El para fomentar el desarrollo cognitivo de los participantes a partir de la práctica de actividad física ${ }^{12}$.

Las propuestas de ApS han sido evaluadas a través de diferentes instrumentos, como entrevistas ${ }^{2}$, cuestionarios o diarios cumplimentados por los estudiantes que presentaban el servicio ${ }^{10}$, aunque también se han evaluado mediante rúbricas de evaluación ${ }^{11}$ o mediante el análisis de la satisfacción de los colectivos que reciben el servicio, es decir, el alumnado y profesorado de los centros de destino ${ }^{10}$. Esto último contribuye a conocer el punto de vista de los diferentes colectivos implicados, lo que permite detectar algunos problemas de implementación de dicha metodología. Con todo ello, el presente estudio analiza la satisfacción de los maestros de Educación Infantil de diferentes centros educativos en los que se había aplicado una propuesta de ApS basada en la educación motriz en la etapa de infantil a partir de la creación de ambientes de aprendizaje durante el tiempo del recreo.

\section{MATERIAL Y MÉTODOS}

\section{Participantes y contexto}

La presente investigación forma parte del proyecto de innovación docente "Implantación y desarrollo del aprendizaje-servicio en las Facultades de Educación de la UCLM", en concreto, de la acción "Ambientes de aprendizaje y recreos activos en Educación Física en Educación infantil" en el que participaron 8 colegios de la ciudad de Cuenca (España) y el alumnado de $3^{\circ}$ de Grado de Maestro en Educación Infantil de la Facultad de Educación de esa misma ciudad. La muestra se divide entre los prestadores del servicio (estudiantes del Grado de Maestro en El), los receptores del servicio (alumnado de la etapa de El participante en la experiencia) y los informantes externos (maestros de El). Sus características se detallan a continuación:

Prestadores del servicio: 46 futuros maestros de El $\left(\mathrm{M}_{\text {edad }}=21,83 \pm 2,16\right.$ años) completaron el proceso ApS. Todos los participantes eran alumnos del Grado de Maestro de Educación Infantil. La muestra se dividía en 44 mujeres y 2 hombres.

Receptores del servicio: 576 alumnos de El de 8 colegios públicos de la ciudad de Cuenca (España) tomaron parte en esta experiencia. Las características de los receptores del servicio se detallan en la Tabla 1. 
Tabla 1. Distribución del alumnado de El participante en la experiencia de ApS.

\begin{tabular}{lcc}
\hline & Niñas & Niños \\
\hline $\mathbf{3}$ años & 77 & 87 \\
$\mathbf{4}$ años & 98 & 95 \\
$\mathbf{5}$ años & 109 & 110 \\
\hline
\end{tabular}

- Informantes externos: 15 maestros $\left(M_{\text {edad }}=47,67 \pm 9,03\right.$ años; $M_{\text {experiencia }}=23,20 \pm 9,82$ años $)$ de los 8 colegios participantes, presentes durante el desarrollo de la actividad, que completaron una escala de valoración de la experiencia. 12 participantes eran mujeres y 3 hombres.

\section{Diseño}

El presente trabajo forma parte de una investigación más amplia, en la que se pretende examinar el efecto del ApS en la formación inicial de los maestros de infantil y primaria, en los receptores del servicio y en el resto de los agentes implicados (profesores y familias). Para este trabajo se pretendía conocer la percepción de los maestros de infantil presentes durante el desarrollo de los recreos activos dinamizados mediante ambientes de aprendizaje, por lo que se realizó una única medida al finalizar la experiencia con un enfoque mixto cuantitativo y cualitativo.

\section{Instrumentos}

Satisfacción de la experiencia ApS-Recreos activos: para conocer la opinión de los maestros presentes durante la realización de los recreos activos se elaboró un cuestionario Ad-hoc compuestos por dos secciones. En la primera se pedía que el profesorado valorará los siguientes aspectos: organización, material, disfrute, participación y novedad, utilizando para ello una escala tipo Likert de 5 puntos (de $1=$ muy mal a $5=$ muy bien). En la segunda sección se planteaban las siguientes preguntas de respuesta abierta: Aspectos positivos $y$ aspectos negativos de la experiencia y ¿Volverías a participar en una experiencia similar? Explica tu respuesta.

\section{Procedimiento}

En el marco de un proyecto de innovación docente, el alumnado de la asignatura de Diseño y desarrollo curricular de Educación Física en Educación Infantil realizó una experiencia ApS dinamizando 9 recreos en 8 colegios públicos de Cuenca (España). Durante el tiempo del recreo (30 minutos) los receptores del servicio (alumnado de educación infantil) podían jugar libremente con el material construido por los prestadores del servicio, que estaba dispuesto en forma de ambientes de aprendizaje. Durante el mes anterior al comienzo de las intervenciones, el alumnado había recibido formación respecto a la metodología de ApS, a la creación de ambientes de aprendizaje y a la construcción de material de EF con material reciclado. 
El grupo-clase fue dividido en 10 grupos, que de-bían crear 2 ambientes de aprendizaje por grupo. A cada grupo se le asignó uno de los siguientes contenidos: desplazamientos, saltos, equilibrio, coordinación óculo-manual y coordinación óculo-pédica.

Los maestros presentes durante el recreo fueron invitados a completar después un cuestionario de evaluación, de forma anónima. Los requisitos eran que al menos debía hacerlo uno de los maestros presentes en cada colegio. El cuestionario se envió por correo electrónico al profesorado, que lo completó en línea de forma anónima utilizando la aplicación Google Forms.

\section{Análisis de datos}

Tras comprobar que los datos no seguían una distribución normal, se aplicaron pruebas no paramétricas para el análisis estadístico de las respuestas del cuestionario. Para comparar las diferencias en las puntuaciones otorgadas a cada factor por el total de los participantes, se aplicó el test de Friedman, utilizando la prueba de los rangos con signo de Wilcoxon como post-hoc.

Los resultados relacionados con las preguntas abiertas se han categorizado en: aspectos relacionados con el diseño y organización de la propuesta, aspectos relacionados con la participación del alumnado y aspectos relacionados con la repercusión de la propuesta. Se expresan los porcentajes de las categorías elaboradas para respuestas más recurrentes en cada una de las preguntas.

\section{RESULTADOS}

En la Tabla 2 se muestran los estadísticos descriptivos $(M \pm D T)$ de la puntuación total otorgada por los participantes a cada una de las variables de análisis: organización, material, disfrute, participación y novedad.

Tabla 2: Estadísticos descriptivos de las variables de satisfacción.

\begin{tabular}{cccccc}
\hline & Organización & Material & Disfrute & Participación & Novedad \\
\hline M \pm DT & $4,67 \pm 0,49$ & $4,40 \pm 0,91$ & $4,67 \pm 0,49$ & $4,60 \pm 0,63$ & $4,47 \pm 0,64$ \\
$\begin{array}{c}\text { Rango } \\
\text { promedio }\end{array}$ & 3,23 & 2,77 & 3,20 & 3,07 & 2,73 \\
\hline
\end{tabular}

El test de Friedman no muestra diferencias significativas entre las diferentes variables $\left(x^{2}=3,544 ; p=0,471\right)$. Sin embargo, se puede observar como en todas las variables la media está muy cercana a 5 , siendo esta la mayor puntuación posible. 


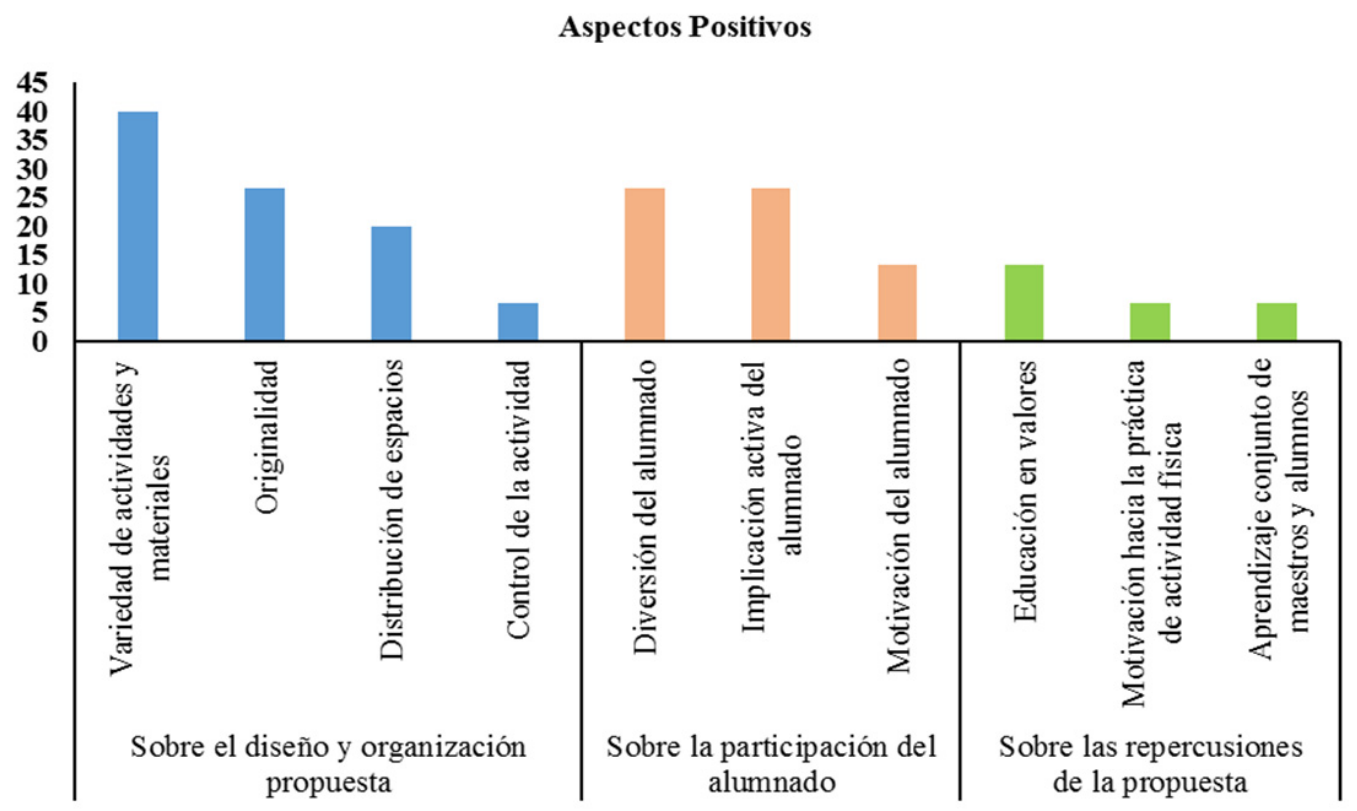

Figura 1. Aspectos positivos de la propuesta. Porcentaje de las respuestas más recurrentes.

Aspectos negativos

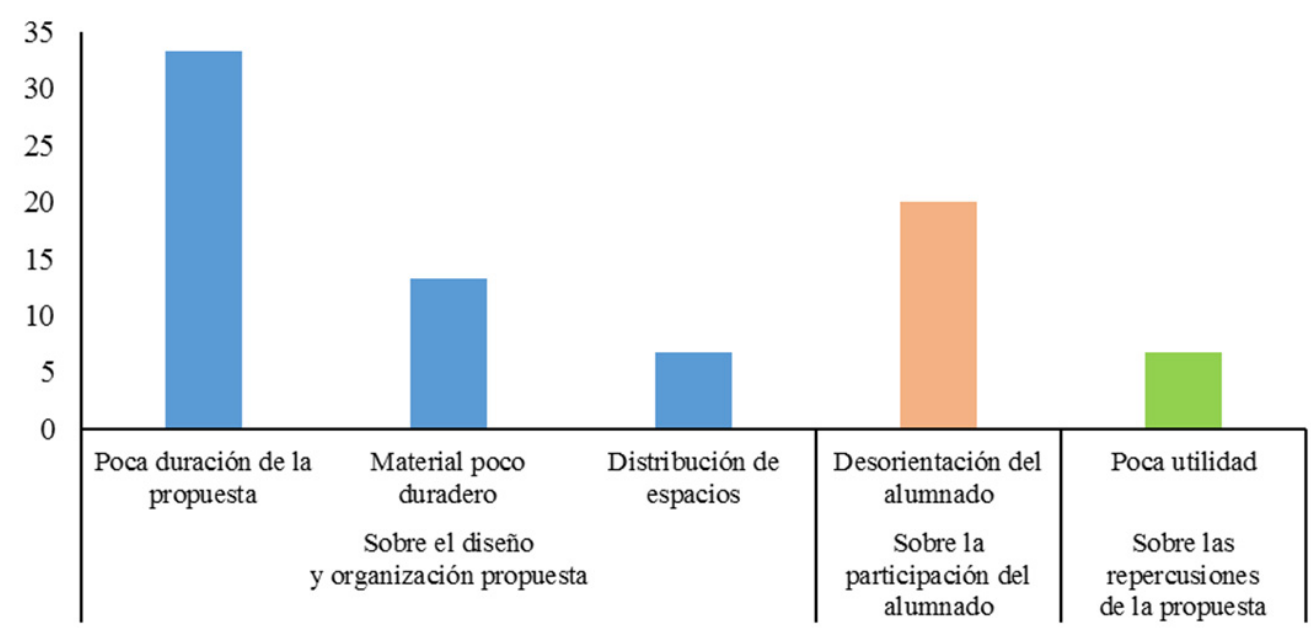

Figura 2: Aspectos negativos de la propuesta. Porcentaje de las respuestas más recurrentes.

En relación a los aspectos positivos sobre el diseño y organización de la propuesta, se pueden destacar afirmaciones como "Mucha imaginación al elaborar tantos juegos con materiales de bajo coste y reciclados" o "La originalidad, Io rápido que organizaron el espacio y los juegos. La concepción de la actividad en si". Respecto a la participación del alumnado, se pueden destacar afirmaciones como "Los alumnos se divierten mucho" o "Como los niños respetaron turnos y jugaron sin decirles aquî. Por último, respecto a las repercusiones de la propuesta, se pueden destacar afirmaciones como "Esta actividad dinamiza el recreo de los más pequeños; Potencia el desarrollo de las relaciones interper- 
sonales y la educación en valores al interactuar los niños de diferentes niveles y con sus compañeros; reduce la agresividad en el juego; motiva a los niños a realizar actividades físicas" o "En ningún momento se pelearon o acercaron a las maestras para quejarse, cosa que habitualmente hacen mucho y sólo juegan a peleas".

En relación a los aspectos negativos sobre el diseño y organización de la propuesta, se pueden destacar afirmaciones como "No ser una actividad puntual, sino realizar esta propuesta con más continuidad a lo largo del curso (al menos una vez a la semana). Podría tener un poco más de duración, una hora de recreo activo" o "Material un poco endeble". Respecto a la participación del alumnado, se pueden destacar afirmaciones como "En algunos juegos los alumnos/as no sabían muy bien qué hacer" o "Desorientación de los niños". Por último, respecto a las repercusiones de la propuesta, se pueden destacar afirmaciones como "En Infantil no le veo tanta utilidad, ya que es como normalmente se trabaja en esta etapa educativa". Cabe destacar que el $20 \%$ de los participantes afirmaron que no encontraban ningún aspecto negativo en la propuesta.

Razones para repetir la experiencia

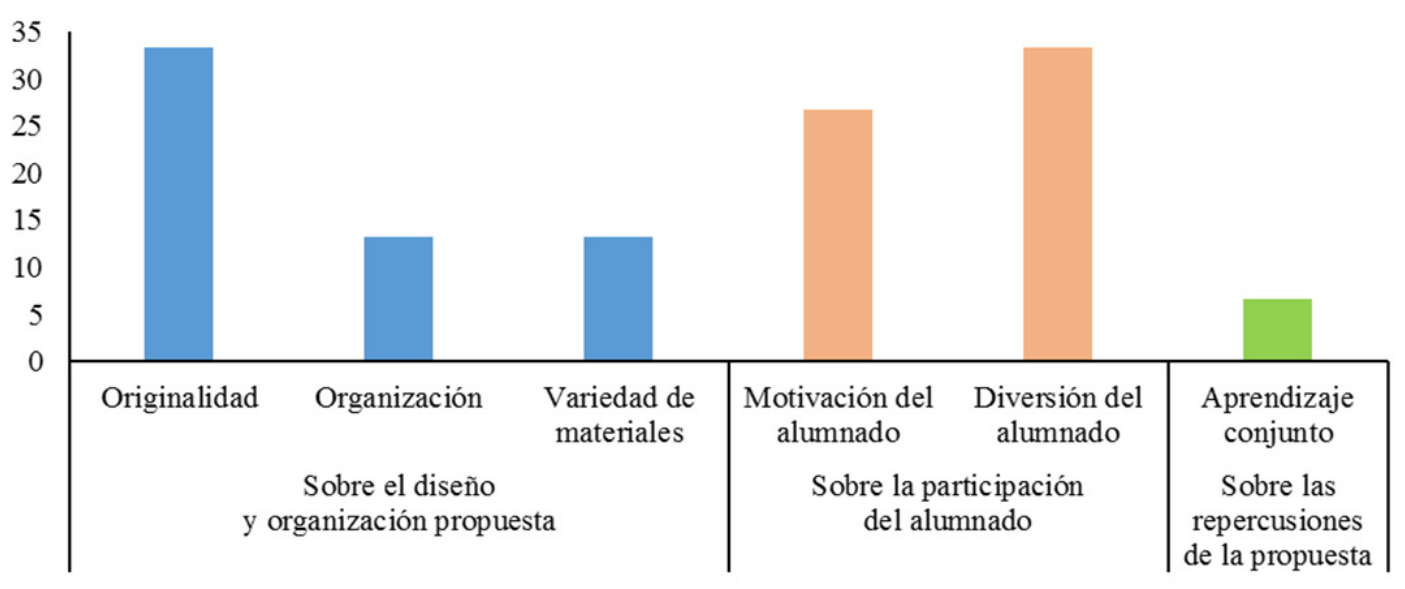

Figura 3. Razones para repetir la propuesta. Porcentaje de las respuestas más recurrentes.

En relación a las razones para volver a repetir la experiencia sobre el diseño y organización de la propuesta se pueden destacar las siguientes afirmaciones: "Porque es una actividad muy organizada y se dispone en un día de mucho material novedoso para todo el alumnado de Infantil" o "Me encantó y me pareció todo muy original". Respecto a la participación del alumnado, se pueden destacar las siguientes a irmaciones: "Porque es algo que en el centro no se realiza y los chicos se lo pasaron genial" o "Me pareció muy interesante y atractiva para los niños". Por último, respecto a las repercusiones de la propuesta se puede destacar la siguiente afirmación: "Siempre vienen bien experiencias nuevas, también los profes aprendemos”. 


\section{DISCUSIÓN}

El objetivo del presente estudio era comprobar el nivel de satisfacción de los maestros de Educación Infantil presentes en una propuesta de ApS. Esta estaba basada en la educación motriz mediante ambientes de aprendizaje en los que se utilizaba material alternativo reciclado. Con este objetivo, los maestros presentes durante el desarrollo de los recreos activos fueron invitados a completar un cuestionario $A d-h o c$ compuesto por una parte cuantitativa y otra cualitativa.

Los resultados revelan que los participantes mostraron altos niveles de satisfacción en todas las variables evaluadas (organización, material, disfrute, participación y novedad), materializados en respuestas muy cercanas a la máxima posible. En términos generales, los resultados se encuentran en consistencia con los expuestos por López-Fernández y Benítez-Porres, en cuyo estudio destacan el agradecimiento de los maestros a los prestadores del servicio, ya que la propuesta fomentaba una mayor participación del alumnado y una mejor organización de las actividades debido a una mayor afluencia de personas de apoyo (prestadores del servicio) ${ }^{10}$.

En esta línea podemos encauzar algunos de los aspectos resaltados como positivos en la propuesta, ya que los docentes manifestaron que los alumnos disfrutaron mucho y participaron activamente y con motivación. Por otra parte, los maestros señalaron que se trataba de una propuesta original, con una gran variedad de actividades y materiales, siendo estos los aspectos más valorados. Por lo tanto, parece que los futuros maestros de El consiguieron crear ambientes de aprendizaje con materiales suficientemente atractivos y provocadores, de acuerdo con lo expuesto por Gil et al. ${ }^{9}$. A la vista de tales resultados, parece interesante destacar el doble servicio prestado por los estudiantes: por un lado la educación motriz de los receptores del servicio y, por el otro, la enseñanza de diferentes actividades realizadas a partir de material reciclado a los docentes.

Algunos maestros también destacaron como aspectos positivos la educación en valores y el aprendizaje conjunto de prestadores y receptores del servicio, aspectos que caracterizan este tipo de metodología y que han sido destacados por numerosos autores ${ }^{11}$.

Por otra parte, como aspectos más negativos de la propuesta, los participantes destacan la poca duración de esta, volviendo a poner en énfasis la satisfacción con la misma y el deseo de que se alargase más en el tiempo. También des-tacan la poca durabilidad de los materiales, aspecto normal debido a que se trata de material reciclado y elaborado por los prestadores del servicio. Por último, algunos maestros destacan que los alumnos estaban desorientados en algunas actividades, ya que no sabían qué hacer, y que hubiera estado bien una mejor clarificación de las actividades por parte de los futuros maestros. En relación con el último aspecto, cabe destacar la que metodología empleada en las actividades se basaba en los modelos comprensivos, por lo que una explicación más exhaustiva de los juegos no hubiera permitido la indagación libre a los alumnos. 
Para finalizar, todos los maestros afirman que repetirían la propuesta, dando como razones para esta decisión los aspectos anteriormente destacados como aspectos positivos de la experiencia.

\section{CONCLUSIONES}

En términos generales, los participantes se sienten satisfechos tanto con el diseño y organización de la propuesta (originalidad, organización y material), como con la participación del alumnado. Por este motivo, todos los maestros afirman que estarían dispuestos a repetir la experiencia, aunque adolecen de una mayor duración de la misma, un material más duradero y, en algunos casos, propuestas más dirigidas.

\section{LIMITACIONES Y CAMINOS FUTUROS}

A continuación, se enumerarán una serie de limitaciones detectadas, que se presentan con sugerencias de mejora para futuras investigaciones. En primer lugar, se presenta un trabajo con naturaleza de experiencia didáctica, por lo que la aplicabilidad de sus resultados a contextos distintos debe ser realizada con cautela. Se sugiere implementar investigaciones en diferentes contextos (por ejemplo: rurales y urbanos; colegios públicos, concertados y privados y diferentes comunidades autónomas) con el objetivo de poder generalizar los hallazgos del presente trabajo. En segundo lugar, dentro del proceso de formación del alumnado de la Facultad de Educación participante, se permitió que realizaran modificaciones a sus materiales entre cada una de las actuaciones, sin modificar el contenido motriz de trabajo. Por ello, los ambientes no han sido exactamente iguales en cada uno de los colegios, lo que se puede considerar una limitación de la investigación. Sin embargo, la riqueza de este tipo de trabajos, utilizando la investigación-acción como medio de aprendizaje, justifican esta decisión. En último lugar, podría haber sido interesante obtener una información más completa de la implementación, con una evaluación del trabajo de los contenidos propuestos. Sin embargo, los participantes, maestros de los colegios implicados, no conocían los contenidos propuestos en cada uno de los ambientes antes de la realización del recreo, por lo que no pudieron ser preguntados sobre ello. Se sugiere para futuras investigaciones informar a los maestros previamente de los contenidos con el objetivo de evaluar el trabajo de contenidos.

\section{REFERENCIAS BIBLIOGRÁFICAS}

1. Ayuste, A, Roig, A, Suari, N, Juanola, M. Aprendizaje-servicio y codiseño en la formación de maestros: vías de integración de las experiencias y perspectivas de los estudiantes. Bordón. Revista de Pedagogía. 2016; 68(2):169-183. https://doi. org/10.13042/Bordon.2016.68211.

2. Baldwin, SC, Buchanan, AM, Rudisill, ME. What teacher candidates learned about 
diversity, social justice, and themselves from service-learning experiences. Journal of TeacherEducation.2007;58(4):315-327.https://doi.org/10.1177/0022487107305259.

3. Bates, AK, Drits, D, Allen, C, McCandless, P. Service learning as an instructional strategy for the preparation of teachers. The Journal of Effective Teaching. 2009; 9:5-23.

4. Blandez, J. La utilización del material y el espacio en Educación Física. Barcelona: Inde. 1995.

5. Capella, C, Gil, J, Martí, M. La metodología del aprendizaje-servicio en la educación física. Apunts. Educación Física y Deportes. 2014; 116(2):33-43. https://doi:10.5672/ apunts.2014-0983.es.(2014/2).116.03.

6. Capella, C, Gil, J, Martí, M, Chiva, O. Estudio de caso múltiple con historias de vida en el grado de educación infantil: aprendizaje-Servicio en la didáctica de la educación física. Profesorado. 2015; 1(19):334-348.

7. Chiva-Bartoll, O, Peris, CC, Piquer, MP. Investigación-acción sobre un programa de aprendizaje-servicio en la didáctica de la educación física. Revista de Investigación Educativa. 2018; 36(1):277-293. https://doi.org/10.6018/rie.36.1.270581.

8. García, T. Aportaciones ciudadanas desde el aprendizaje servicio: universidad, escuela y comunidad conectadas. Revista Interuniversitaria de Formación del Profesorado. $2011 ; 25(2): 125-141$.

9. Gil, L, Guzmán, M, Moreno, E. Caminando hacia la escuela que queremos. Barcelona: Rosa Sensat-Octaedro. 2013.

10. López-Fernández, I, Benítez-Porres, J. El Aprendizaje Servicio en la Universidad: una experiencia en el marco de una asignatura del Grado en Educación Primaria. REDU. Revista de Docencia Universitaria. 2018; 16(2):195-210.

11. Martínez, A. El aprendizaje servicio como experiencia en el Grado de Educación Primaria. RIDAS. Revista Iberoamericana de Aprendizaje Servicio. 2018; 5:142-149.

12. Nielsen, A, Romance, AR, Chichilla, JL. Los ambientes de aprendizaje como metodología activa promotora de la actividad física en Educación Infantil. Un estudio de caso. Retos. 2020; 37(37):498-504.

13. Riera, MA, Ferrer, M, Ribas, C. La organización del espacio por ambientes de aprendizaje en la Educación Infantil: significados, antecedentes y reflexiones. RELAdEI. Revista Latinoamericana de Educación Infantil. 2014; 3(2):19-39.

14. Vázquez, S, Liesa, M, Lozano, A. Recreos cooperativos e inclusivos a través de la metodología de Aprendizaje-Servicio. Revista Electrónica Interuniversitaria de Formación del Profesorado. 2017; 20(1):173-185. http://doi:10.6018/reifop/20.1.213181. 